\title{
Rationale for the design of a novel tool for immunotherapy based on an emulsion of glycosaminoglycan
}

\author{
Marco Ruggiero* and Stefania Pacini \\ Silver Spring Sagl, Switzerland
}

\begin{abstract}
In this article, we describe the rationale behind the design of an innovative immunotherapeutic tool designated imuno ${ }^{\mathrm{TM}}$ that is an emulsion of microbial, lowmolecular-weight chondroitin sulfate non-covalently bound to pure phosphatidylcholine with vitamin $\mathrm{D}_{3}$ intercalated in the ensuing multi-molecular structure. The rationale for this design derives from the decade-old observation that chondroitin sulfate is the active principle responsible for the immune-stimulatory, anti-cancer properties of cartilage extracts and from the more recent observation that a known immunotherapeutic compound, the Gc protein-derived Macrophage Activating Factor (GcMAF), works as an adjuvant similar, in principle, to the incomplete Freund's adjuvant. From these premises, we designed an innovative tool that uses an ultrapure, low-molecular-weight chondroitin sulfate, thus overcoming all limitations associated with blood-derived or animal-derived non-ultrapure extracts, at the same time guaranteeing constant sulfation profile and charge density. This novel form of chondroitin sulfate is non-covalently bound to pure phosphatidylcholine in a self-assembly manner that is coherent with the principles of negentropy. Vitamin $\mathrm{D}_{3}$ is then intercalated in this structure that resembles protocells made of a phosphatidylcholine bilayer. Such an association reduces the main side effect of vitamin $\mathrm{D}_{3}$ that is the induction of hypercalcemia, at the same time maximizing its immunotherapeutic potential. In addition, we describe the mechanism of action of this immunotherapeutic tool with particular reference to stimulation of innate immunity through a mechanism shared by adjuvants such as Freund's adjuvant. Finally, we describe an example of a transdermal delivery system and we discuss possible applications.
\end{abstract}

\section{Immunotherapy with glycosaminoglycans}

The term "immunotherapy" encompasses a wide number of treatments that harness the immune system to fight diseases ranging from cancer [1] to asthma [2] and Alzheimer's disease [3]. Although this approach has been implemented with success in a wide variety of diseases and conditions, immunotherapy of cancer appears to yield the most promising results, and it is now considered an integral component of the armamentarium for the treatment of patients with advanced tumors [1]. Both the cellular and the humoral components of the immune system are targets of immunotherapeutic approaches as well as the innate and adaptive arms of the system, thus leading to an extraordinarily high number of remedies and strategies that differ in terms of specificity, selectivity, and efficacy. Among the different types of immunotherapy, we recently focused our attention on those approaches that are based on glycosaminoglycans with particular reference to the work of Dr. Prudden [4]. Since 1957, Prudden and Colleagues described how extracts from bovine cartilage exerted a plethora of therapeutic effects that today would be described as immunotherapy, ranging from accelerated wound healing [5], to decrease of inflammation [6], and successful treatment of advanced cancers [4]. In this specific context, in 1985, Prudden wrote "Oral and subcutaneous administration of specific preparations of bovine tracheal cartilage rings (Catrix), a nontoxic agent, has resulted in a high response rate in 31 cases of a variety of clinical malignancies (response rate $90 \%, 61 \%$ complete). The demonstrated responder includes present therapeutic disasters such as glioblastoma multiforme and cancers of the pancreas and lung. Other types which were treated with success included cancers of the ovary, rectum, prostate, cervix, thyroid, and an inoperable squamous cancer of the nose." [6]. Three years later, in 1988, Prudden and Colleagues were able to identify the molecule responsible for the successful treatment of those "present therapeutic disasters" and concluded that chondroitin sulfate, a glycosaminoglycan, was responsible for what they called "immunoaugmenting activity" of the bovine cartilage extract [7].

Dr. Prudden passed away the same year he identified chondroitin sulfate as the active agent of his preparation; his work was well described in layman's terms in the obituary published in The New York Times that reads "Dr. Prudden also reported research that used cartilage treatment to help osteoarthritis patients and he believed that it could be used to treat cancer and other diseases." [8]. The results observed by Prudden and Colleagues have been independently replicated, and a recent paper published in 2018 by researchers working at Faculty of Medicine of the American University of Beirut, Lebanon, demonstrates that "bovine cartilage induces tumor cell death through apoptosis and this mechanism of action is the same across different cell lines and species." [9] thus supporting the notion that chondroitin sulfate exerts general anti-cancer effects not limited to a specific histologic type. Such a universal anticancer effect can be explained by assuming that chondroitin sulfate works on multiple types of immune cells, most notably on cells pertaining to the innate arm of the immune system.

Consistent with these observations, in 2016 we wrote that chondroitin sulfate, in addition to being the active molecule responsible for the anticancer effects of cartilage, was also the molecule responsible

${ }^{\star}$ Correspondence to: Marco Ruggiero, Silver Spring Sagl, Via Raimondo Rossi 24, Arzo-Mendrisio 6864, Switzerland, Tel: +41 79230 9283, E-mail: marco.drruggiero@gmail.com

Key words: autism, cancer, GcMAF, immunotherapy, vitamin D

Received: August 06, 2018; Accepted: August 23, 2018; Published: August 27 2018 
for the immunotherapeutic effects of an elusive compound termed Gc protein-derived Macrophage Activating Factor (GcMAF) [10]. Such a concept stemmed from more than thirty years of research in the field of glycosaminoglycans and from the observation that chondroitin sulfate is composed of repetitive units of alternating sugars that are glucuronic acid and $\mathrm{N}$-acetyl-galactosamine, the latter being the active site of GcMAF [11]. Shortly after publication of our paper, this concept was independently confirmed by the observation that chondroitin sulfate inhibits angiogenesis in the chick embryo chorioallantoic membrane [12] that is another anticancer property attributed to GcMAF that we had previously described [13]. In addition, it has been recognized for a long time that heparin, a glycosaminoglycan with a molecular structure closely related to chondroitin sulfate, inhibits cancer cell proliferation [14], even though the anticoagulant effects of this molecule prevent its use as a bona fide anticancer treatment. It is worth noticing, however, that the anticancer effects of heparin are independent of its anticoagulant activity, and a recent study demonstrated how low-molecular-weight heparin may represent a novel therapeutic approach to the prevention of metastases thanks to its effects on the endothelial barrier that are not associated with the anticoagulant activity [15].

Based on these facts and consideration, in 2015 we formulated an emulsion of chondroitin sulfate, vitamin $\mathrm{D}_{3}$ and oleic acid that was intended to modulate the immune system and reproduce the biological and clinical effects attributed to GcMAF. Use of this compound, designated Rerum ${ }^{\circledR}$, yielded encouraging results in the complementary approach to a number of conditions ranging from cancer to autism, to acute and chronic pain [16-18]. Here we describe the rationale for the design of a novel tool for immunotherapy based on our previous experience with glycosaminoglycans, GcMAF and Rerum ${ }^{\circledR}$.

\section{A novel type of chondroitin sulfate}

Until recently, chondroitin sulfate was extracted and purified from animal cartilage; because of this, animal-derived chondroitin sulfate is a heterogeneous mixture of high and low-molecular-weight species, with high-molecular-weight species being prevalent, and shows a heterogeneous sulfation profile. Such heterogeneity is well recognized up to the point that the last Revision Bulletin of the United States Pharmacopoeia [19] states that chondroitin sulfate preparations are allowed to have quantities of the glycosaminoglycan ranging from $90 \%$ to $105 \%$ calculated on the dried basis. In other words, a purity of merely $90 \%$ is accepted. In addition, since the process of extraction cannot have a $100 \%$ yield and presence of contaminants has to be accepted, the same document states that protein contamination up to $6 \%$ has to be accepted. Likewise, the presence of heavy metals up to $20 \mathrm{ppm}$ is accepted as well as the presence of non-specific disaccharides up to $10 \%$. In short, animal-derived chondroitin sulfate is a heterogeneous mixture comprising non-negligible amounts of contaminants. A major breakthrough in chondroitin sulfate research was represented by the recent availability of non-animal chondroitin sulfate, a compound that overcomes the limitations of animal-derived compounds as described above. Such chondroitin sulfate is the result of microbial fermentation with the production of unsulfated chondroitin that undergoes siteselective sulfation, modulated in order to obtain a sulfation profile superimposable to that of human chondroitin sulfate. This patented procedure leads to a product that is $99 \%$ pure, has a percentage of typical disaccharides almost identical to that of human synovial fluid and, most importantly, shows consistent low molecular weight with constant charge density and molecular mass parameters [20]. These features represent a significant improvement over animal-derived chondroitin sulfate and such an improvement leads to significantly increased clinical efficacy. Thus, in a recent study, the pharmacokinetic profile of an oral form of this non-animal, low-molecular-weight chondroitin sulfate was compared to that of animal (bovine)-derived chondroitin sulfate in healthy volunteers. Microbial low-molecular-weight chondroitin sulfate yielded a much greater plasma concentration with increased specific sulfation in the 6-position of $\mathrm{N}$-acetyl-galactosamine in human plasma and, as a consequence, the overall charge density was doubled compared to animal-derived chondroitin sulfate [20]. Such a greater efficacy bears important consequences as far as the known anti-inflammatory, "immunoaugmenting" (to quote Dr. Prudden's words) activities of chondroitin sulfate are concerned. A recent study demonstrated how the greater therapeutic efficacy of microbial chondroitin sulfate was mainly due to its homogeneous low-molecularweight. In an animal model of polyarthritis, low-molecular-weight chondroitin sulfate reduced the arthritic score with significantly greater efficiency than high-molecular-weight chondroitin sulfate and, at the same time, decreased gamma-glutamyltransferase activity, plasmatic C-reactive protein levels plasmatic levels of C-reactive protein and the pro-inflammatory cytokines IL-1beta and IL-6 [21]. In summary, microbial low-molecular-weight chondroitin sulfate is a demonstrated significant improvement over animal-derived chondroitin sulfate as it shows much more efficient pharmacokinetics and therapeutic efficacy. Because of these features, we decided to design our next immunotherapeutic tool on the backbone of ultra-pure, homogeneous low-molecular weight chondroitin sulfate that represents a different chemical moiety as compared with animal-derived high-molecularweight chondroitin sulfate.

\section{Design of imuno ${ }^{\mathrm{TM}}$ a multi-molecular complex based on low-molecular-weight chondroitin sulfate, phosphati- dylcholine and vitamin $D_{3}$}

The rationale for the design of imuno ${ }^{\mathrm{TM}}$ lays in the observation that glycosaminoglycans in human blood are assembled in multi-molecular complexes comprising phosphatidylcholine, the major constituent of cell membranes. We first observed such an association between phosphatidylcholine and a human blood glycosaminoglycan, heparin, in 1985 when we demonstrated that circulating endogenous human heparin is complexed with phosphatidylcholine in human plasma [22]. In this paper, we described for the first time the two major features of the physiological phosphatidylcholine/glycosaminoglycan interaction that, 33 years later, led to the design of imuno ${ }^{\mathrm{TM}}$.

1. The glycosaminoglycan, a highly hydrophilic molecule, interacts with phosphatidylcholine, an amphipatic molecule constituting the lipid bilayer of cell membranes, through electrostatic interactions between the negatively charged quaternary ammonium groups of its polar head and the sulfate groups of the glycosaminoglycan, with carboxylic acids possibly contributing to electrostatic stabilization. Such an interaction at the plasma level could represent a mechanism of abstraction and storage of a highly active compound.

2. In addition to this mechanism, we demonstrated how the glycosaminoglycan participated in cell signaling by interacting with phosphatidylcholine of the external layer of the membrane, thus modulating transmembrane second messenger formation through polyphosphoinositide metabolism with the formation of calcium-mobilizing inositol 1,4,5-trisphosphate, and protein kinase C-activating diacylglycerol $[22,23]$.

These observations of ours have been recently confirmed and extended as it appears that the supra-molecular assembly we described 
for human endogenous circulating heparin is a general physiological phenomenon that involves other glycosaminoglycans and, in particular, chondroitin sulfate. In a study published in 2016 , it was demonstrated that chondroitin sulfate interacts mainly with the polar groups of phosphatidylcholine in an experimental cell membrane system exhibiting a dynamic adsorption-desorption process. Interestingly, the electrostatic interaction that we had described in 1985 between the polar head of phosphatidylcholine and the sulfated groups of the glycosaminoglycan, proved instrumental in inducing a higher degree of order in the phosphatidylcholine monolayer mimicking the external layer of the cell membrane as assessed by polarization-modulated infrared reflection absorption spectroscopy [24]. In other words, it appears that the phosphatidylcholine/glycosaminoglycan interaction represents a notable example of negentropy that is consistent with the concept delineated by Nobel Laureate Erwin Schroedinger in 1944 [25], and elaborated in a more recent publication where is reported that "... such an occurrence of molecular self-assembly in the absence of genetic information is thought to have been the first step in the origin of life ... molecules not pertaining to the flow of information in the classical interpretation of molecular biology since the times of Watson and Crick (DNA $\rightarrow$ RNA $\rightarrow$ Proteins) are assembled in our blood following a pattern as archetypical as life itself ..." [26]. The concept that glycosaminoglycans help to establish a higher order, or negentropy, in biological structures similar to cell membranes is further corroborated by results reported in a study authored by researchers working at the Department of Pharmaceutical Sciences of the North Dakota State University. In a paper entitled "Glycosaminoglycan-mediated selective changes in the aggregation states, zeta potentials, and intrinsic stability of liposomes" the Authors describe how the organizational states of liposome bilayers are stabilized by the presence of glycosaminoglycans [27]. This observation is consistent with a more recent paper by Mantovani, et al. (2016) who describe the assembly of endogenous chondroitin sulfate in human blood drawing a distinction between plasma and whole blood, and highlight the role of chondroitin sulfate associated with circulating cells of the immune system where the sulfation profile appears essential for such an association, paralleling what we had described in 1985 [22,28]. Interestingly, the sulfation profile of immune cell-associated chondroitin sulfate in human blood is more similar to that of microbial low-molecular-weight chondroitin sulfate than to that of its animal-derived counterpart with particular reference to specific sulfation in the 6-position of N-acetyl-galactosamine [20]. These data were not available in 2015 when Rerum ${ }^{\circledR}$ was designed, and it is based on these data and the consideration outlined above that we decided to formulate an immune-modulating supra-molecular complex using microbial low-molecular-weight chondroitin sulfate as the backbone of the novel multi-molecular complex termed imuno ${ }^{\mathrm{TM}}$. In imuno ${ }^{\mathrm{TM}}$, pure phosphatidylcholine is bound to microbial low-molecular-weight chondroitin sulfate through non-covalent, electrostatic interactions between the negatively charged quaternary ammonium groups of its polar head and the sulfate groups with particular reference to sulfated $\mathrm{N}$-acetyl-galactosamine that is the active site of GcMAF [11]. Considering that the sulfation profile of microbial low-molecularweight chondroitin sulfate is homogeneous, at variance with its animalderived counterpart, the interactions are more stable, thus favoring a higher level of negentropy that may be superimposable to that observed in the natural assembly of these molecules in the cells of the immune system. In essence, each molecule of microbial low-molecularweight chondroitin sulfate is coated/surrounded by a high number of molecules of phosphatidylcholine that expose their hydrophilic tails.
In an aqueous environment, this prompts the spontaneous formation of cell membrane-like structures under the form of a homogeneous, single-phospholipid bilayer that resembles protocellular structures with chondroitin sulfate mimicking the role of nucleic acids [29].

It is worth mentioning that the formation of ordered structures made of microbial low-molecular-weight chondroitin sulfate and phosphatidylcholine leads to interesting consequences as far as alternative biological signaling is concerned. Both chondroitin sulfate and phosphatidylcholine are highly charged molecules that are propelled at speeds varying from $40 \mathrm{~cm} / \mathrm{s}$ in the aorta to $0.03 \mathrm{~cm} / \mathrm{s}$ in capillaries, thus showing positive and negative acceleration at every heartbeat. Acceleration of an electric charge is invariably associated with an electromagnetic field that, in the case of the structures described above, it is a varying field whose frequency and wavelength depend on a number of variables that are strictly individual such as, for example, heart frequency, hemodynamics, factors of turbulence, and so on. This varying electromagnetic field can be interpreted as a radio signal, and it is tempting to speculate that it may lead to non-chemical signaling between molecules in circulating blood and DNA. Thus, we recently described how DNA, functioning as a fractal antenna, is able to send and receive signals under the form of radio waves and how this may, in turn, regulate gene expression and, ultimately, epigenetics [30]. Since the assembly of microbial low-molecular-weight chondroitin sulfate and phosphatidylcholine is reminiscent of archetypical protocells or, in other words, of structures directly entangled with the origin of life on earth, the radio signal associated with these structures may be considered one of the most fundamental in biologic signaling and it can be conceived that it helps in restoring gene expression, in particular when it is deranged as it is in the case, for example, of cancer cells.

Vitamin $\mathrm{D}_{3}$ another component of the multi-molecular complex here described, is a secosteroid hormone with such a number of health effects that a review on this topic was provocatively entitled "Does vitamin D make the world go 'round'?" [31]. We have extensively written on the vitamin $\mathrm{D}$ axis, a functional signaling pathway that comprises the vitamin D-binding protein, the precursor of GcMAF, vitamin $\mathrm{D}$ and its protein receptor (VDR) [32]. We have also proposed the molecular mechanism of interaction between GcMAF and VDR as it derives from the observation that biological responses to GcMAF depend on VDR polymorphisms [33,34]. In the formulation of imuno ${ }^{\mathrm{TM}}$ vitamin $\mathrm{D}_{3}$ is incorporated in the protocellular structures described above in a manner similar to that described for liposomes made of phosphatidylcholine [35]. It is worth mentioning that the incorporation of vitamin $\mathrm{D}_{3}$ in liposomes is a strategy to reduce its main side effect that is hypercalcemia [35]. Since incorporation rate of vitamin $\mathrm{D}_{3}$ in phosphatidylcholine liposomes is more than $80 \%$, imuno $^{\mathrm{TM}}$ may represent a delivery system much more efficient than the Gc protein/GcMAF where only one molecule of vitamin $\mathrm{D}_{3}$ is bound to one molecule of the protein.

In summary, imuno ${ }^{\mathrm{TM}}$ is a multi-molecular complex made of molecules endowed with known healthy properties arranged in such a way as to reproduce archetypical protocells able to send putatively restorative radio signals to DNA. Due to its peculiar molecular design, the function of imuno ${ }^{\mathrm{TM}}$ in the context of immunotherapy shows a dual nature. On one side, the known health effects of its components are amplified by their assembly in a multi-molecular structure held together by a higher number of non-covalent bonds. On the other side, the physical-chemical features of the emulsion enable imuno ${ }^{\mathrm{TM}}$ to function in a manner superimposable to that of Freund's adjuvants. 


\section{Mechanism of action of imuno ${ }^{\mathrm{TM}}$ as a Freund's adjuvant, indications and ways of administration}

The first description of what is now called Freund's adjuvant can be found in a publication of 1942 where Freund and McDermott described a method of immunization in which the antigen - in that case, horse serum - was emulsified in an oily suspension of heat-inactivated dried mycobacteria. According to their results, a single injection of this emulsion resulted in the development of large amounts of antibodies. After this first report, several publications described the efficacy of this approach in inducing a strong immunological response against a wide variety of antigens and, as of today, there are close to 13,000 peer-reviewed papers listed in PubMed describing the use of Freund's adjuvant. Among these publications, it is worth mentioning a freely available review written in 1955 that provides an interesting historical perspective on the matter. Freund's adjuvant exists in two forms, the complete one that comprises inactivated dried mycobacteria (usually Mycobacterium tuberculosis), and the incomplete form that is only an emulsion of water and mineral oil. Although Freund's adjuvant was originally intended to boost immune responses, numerous other fields of application have been proposed ranging from diabetes [36] to Parkinson's disease [37]. As far as cancer is concerned, Freund's adjuvant has been proposed as an effective tool in cancer vaccines [38] and encouraging results using the incomplete form of the adjuvant have been reported in patients with melanoma [39]. The main limitations of the original Freund's adjuvant are associated with the use of mineral oil and, for this reason, modern versions of the adjuvant use other types of lipophilic compounds such as squalene or emulsifying agents such as polyacrylic acid [40] thus supporting the concept that it is the physical nature of the emulsion rather than its chemical composition responsible for the stimulation of the immune system. Such a concept is corroborated by the observation that adjuvants induce a variety of immunological responses ranging from synthesis of cytokines and chemokines to recruitment of immune cells and induction of antigen transport to draining lymph nodes. It is accepted that adjuvants primarily stimulate the innate arm of the immune system by favoring a local immuno-competent environment at the site of injection [41]. Since the innate arm of the immune system comprises cell types as different as macrophages, neutrophils, dendritic cells, natural killer cells and mast cells as well as cytokines and molecules of the complement system, stimulation of innate immunity by emulsions such as imuno ${ }^{\mathrm{TM}}$ provides a much wider spectrum of immunotherapeutic targets as compared with molecules that activate only one type of cells of the immune system. From the standpoint of a more recent historical perspective, it is interesting to notice that also GcMAF was originally proposed by Yamamoto as an adjuvant for antibody production [42], and it is likely that such an activity is indeed responsible for a number of the effects that have been observed in fields where macrophage activation and immune deficiency play little, if any, role such as autism [43]. In addition, the ascription of the clinical effects of GcMAF to its adjuvant activity would solve many of the inconsistencies that we have described in a recent paper [10]. The adjuvant activity of GcMAF is consistent with the molecular models we recently proposed [34], and may explain the greater efficacy of a GcMAF derivative we invented, where oleic acid was non-covalently bound to the protein [44]. imuno ${ }^{\mathrm{TM}}$, at variance with GcMAF and Rerum $^{\circledR}$, contains neither blood-derived proteins nor animal-derived components that may interfere with features of the emulsion that are required to exert maximal adjuvant activity without side effects. In addition, the peculiar molecular design of imuno ${ }^{\mathrm{TM}}$ enables ways of administration that add great versatility to its use and enable a wide spectrum of indications.
Thus, because of its composition, imuno ${ }^{\mathrm{TM}}$ can be manufactured as a sterile emulsion for subcutaneous injection that exploits its adjuvant activity. It is worth noticing that such a way of administration was recognized by Prudden as the most effective for bovine cartilage extracts containing chondroitin sulfate [4], and subsequent experiences with Rerum $^{\circledR}$ by a number of therapists confirm this assumption [18]. In addition, imuno ${ }^{\mathrm{TM}}$ can be compounded under the form of a transdermal delivery system following the concepts that we have recently described in a paper dealing with optimization of HIV DNA vaccines [45], an area where stimulation of the innate immune system may provide significant results. Compounding of imuno ${ }^{\mathrm{TM}}$ under the form of a transdermal delivery system (cream) would increase the spectrum of indications that in addition to those recognized for GcMAF and Rerum ${ }^{\circledR}$ would thus comprise dermatologic and rheumatologic conditions where the rebalancing of the immune system is sought after. In addition, since the system that we have previously described significantly increases transdermal delivery, it can be foreseen that imuno ${ }^{\mathrm{TM}}$ may yield effects at the site of application not too different from those observed with injection. In short, the field of application of imuno ${ }^{\mathrm{TM}}$ comprises, but it is not limited to, all conditions and diseases that have been successfully targeted by GcMAF and Rerum ${ }^{\circledR}$ ranging from cancer to autism, acute and chronic pain, autoimmune diseases and inflammatory bowel disease $[17,18,43,46,47]$.

In conclusion, design and development of imuno ${ }^{\mathrm{TM}}$ represents the culminating point of research in the field of glycosaminoglycans begun in the early eighties as well as the result of years of achievements in the field of immunotherapy with particular reference to GcMAF and Rerum $^{\circledR}$

\section{Authorship and contributorship}

Marco Ruggiero and Stefania Pacini developed the concepts described in this paper and contributed equally to this manuscript.

\section{Competing interests}

Marco Ruggiero is the founder and CEO of Silver Spring, a Swiss company dedicated to research, development, and production of supplements and probiotics. No product of Silver Spring is mentioned in this article. Marco Ruggiero has invented a number of products including the product designated imuno ${ }^{\mathrm{TM}}$ here described and consults for several companies.

Stefania Pacini has invented the product designated imuno ${ }^{\mathrm{TM}}$ here described and consults for several companies.

\section{Advisory}

No information in this paper is presented by the authors as medical advice. Caregivers, researchers and interested parties should research all information given. Beginning any significant biomedical or other interventions that may impact physiology or making changes to an established regimen should be discussed with the patient's physician in advance. Standard of care for each pathology must be followed as well as rules and regulations established by Health Authorities of each Country.

\section{References}

1. Saied A, Pillarisetty VG, Katz SC (2014) Immunotherapy for solid tumors--a review for surgeons. J Surg Res 187: 525-535. [Crossref]

2. Erekosima N, Suarez-Cuervo C, Ramanathan M, Kim JM, Chelladurai Y, et al. (2014) Effectiveness of subcutaneous immunotherapy for allergic rhinoconjunctivitis and asthma: a systematic review. Laryngoscope 124: 616-627. [Crossref] 
3. Delrieu J, Ousset PJ, Voisin T, Vellas B (2014) Amyloid beta peptide immunotherapy in Alzheimer disease. Rev Neurol (Paris) 170: 739-748. [Crossref]

4. Prudden JF (1985) The treatment of human cancer with agents prepared from bovine cartilage. J Biol Response Mod 4: 551-584. [Crossref]

5. PRUDDEN JF, NISHIHARA G, BAKER L (1957) The acceleration of wound healing with cartilage. I. Surg Gynecol Obstet 105: 283-286. [Crossref]

6. Prudden JF, Balassa LL (1974) The biological activity of bovine cartilage preparations. Clinical demonstration of their potent anti-inflammatory capacity with supplementary notes on certain relevant fundamental supportive studies. Semin Arthritis Rheum 3: 287-321. [Crossref]

7. Rosen J, Sherman WT, Prudden JF, Thorbecke GJ (1988) Immunoregulatory effects of catrix. J Biol Response Mod 7: 498-512. [Crossref]

8. (1998) NY Times ARCHIVE. John F. Prudden, 78, Surgeon and Researcher. By Ford Burkhart.

9. Tanelian A, Abdelnoor AM (2018) The Action of Bovine Cartilage on Tumor Cells In Vitro And In Vivo. Am J Immunol.

10. Ruggiero M, Reinwald H, Pacini S (2016) Is chondroitin sulfate responsible for the biological effects attributed to the GC protein-derived Macrophage Activating Factor (GcMAF)? Med Hypotheses 94: 126-131. [Crossref]

11. Saburi E, Saburi A, Ghanei M (2017) Promising role for Gc-MAF in cancer immunotherapy: from bench to bedside. Caspian J Intern Med 8: 228-238. [Crossref]

12. Kobayashi T, Kakizaki I, Nozaka H, Nakamura T (2016) Chondroitin sulfate proteoglycans from salmon nasal cartilage inhibit angiogenesis. Biochem Biophys Rep 9: 72-78. [Crossref]

13. Pacini S, Morucci G, Punzi T, Gulisano M, Ruggiero M (2011) Gc protein-derived macrophage-activating factor (GcMAF) stimulates cAMP formation in human mononuclear cells and inhibits angiogenesis in chick embryo chorionallantoic membrane assay. Cancer Immunol Immunother 60: 479-85. [Crossref]

14. Vannucchi S, Pasquali F, Chiarugi VP, Ruggiero M (1991) Heparin inhibits A431 cel growth independently of serum and EGF mitogenic signalling. FEBS Lett 281: 141144. [Crossref]

15. Kevane B, Egan K, Allen S, Maguire P, Neary E, et al. (2017) Endothelial barrier protective properties of low molecular weight heparin: A novel potential tool in the prevention of cancer metastasis? Res Pract Thromb Haemost 1: 23-32. [Crossref]

16. Schwalb M, Taubmann M, Hines S, Reinwald H, Ruggiero M (2016) Clinical Observation of a Novel, Complementary, Immunotherapeutic Approach based on Ketogenic Diet, Chondroitin Sulfate, Vitamin D3, Oleic Acid and a Fermented Milk and Colostrum Product. Am J Immunol 12: 91-98.

17. Antonucci N, Pacini S, Ruggiero M (2017) Clinical Case Report of a Nove Immunotherapeutic Approach to Autism Spectrum Disorders based on an emulsion of Chondroitin Sulfate, Vitamin D3 and Oleic Acid. Am J Immunol 13: 180-185.

18. Peter T, Ruggiero M (2017) Ketogenic Diet and Immunotherapy in Cancer and Neurological Diseases. 4th International Congress on Integrative Medicine, 1, 2 April 2017, Fulda, Germany. Am J Immunol 13: 158-164.

19. https://www.uspnf.com/sites/default/files/usp_pdf/EN/USPNF/revisions/chondroitinsulfate-sodium.pdf

20. Volpi N, Mantovani V, Galeotti F, Bianchi D, Straniero V, et al. (2018) Ora Bioavailability and Pharmacokinetics of Nonanimal Chondroitin Sulfate and Its Constituents in Healthy Male Volunteers. Clin Pharmacol Drug Dev. [Crossref]

21. Bauerova K, Ponist S, Kuncirova V, Drafi F, Mihalova D, et al. (2014) Effect of nonanimal high- and low-molecular-mass chondroitin sulfates produced by a biotechnological process in an animal model of polyarthritis. Pharmacology 94: 10914. [Crossref]

22. Vannucchi S, Ruggiero M, Chiarugi V (1985) Complexing of heparin with phosphatidylcholine. A possible supramolecular assembly of plasma heparin. Biochem J 227: 57-65. [Crossref]

23. Ruggiero M, Fedi S, Bianchini P, Vannucchi S, Chiarugi V (1984) Molecular events involved in the proaggregating effect of heparin on human platelets. Biochim Biophys Acta 802: 372-377. [Crossref]

24. Ceridório LF, Caseli L, Oliveira ON Jr (2016) Chondroitin sulfate interacts mainly with headgroups in phospholipid monolayers. Colloids Surf B Biointerfaces 141: 595-601. [Crossref]

25. (1944) What is life? Erwin Schrodinger. [http://www.whatislife.ie/downloads/What-isLife.pdf]
26. Ruggiero M (2017) Is Rerum ${ }^{\circledR}$ the New Coley's Vaccine? Am J Immunol 13: 91-98.

27. Nyren-Erickson EK, Haldar MK, Totzauer JR, Ceglowski R, Patel DS, et al. (2012) Glycosaminoglycan-mediated selective changes in the aggregation states, zeta potentials, and intrinsic stability of liposomes. Langmuir 28: 16115-16125. [Crossref]

28. Mantovani V, Galeotti F, Maccari F, Volpi N (2016) Analytical Methods for Assessing Chondroitin Sulfate in Human Plasma. J AOAC Int 99: 333-341. [Crossref]

29. Yanagawa H, Ogawa Y, Kojima K, Ito M (1988) Construction of protocellular structures under simulated primitive earth conditions. Orig Life Evol Biosph 18: 179207. [Crossref]

30. (2015) Ruggiero M, Aterini S. Electromagnetic Fields. In: Schwab M. (Edtr) Encyclopedia of Cancer. Springer, Berlin, Heidelberg. [https://www.springer.com/gp/ book/9783540476481]

31. Wagner CL, Taylor SN, Hollis BW (2008) Does vitamin D make the world go 'round'? Breastfeed Med 3: 239-250. [Crossref]

32. Ruggiero M, Pacini S (2011) The vitamin D axis in chronic kidney disease: state of the art and future perspectives. Eur Nephrol 5: 15-19.

33. Pacini S, Morucci G, Punzi T, Gulisano M, Ruggiero M, et al. (2012) Effect of paricalcitol and GcMAF on angiogenesis and human peripheral blood mononuclear cell proliferation and signaling. J Nephrol 25: 577-581. [Crossref]

34. Thyer L, Ward E, Smith R, Fiore MG, Magherini S, et al. (2013) A novel role for a major component of the vitamin $\mathrm{D}$ axis: vitamin $\mathrm{D}$ binding protein-derived macrophage activating factor induces human breast cancer cell apoptosis through stimulation of macrophages. Nutrients 5: 2577-2589. [Crossref]

35. Merz K, Sternberg B (1994) Incorporation of vitamin D3-derivatives in liposomes of different lipid types. J Drug Target 2: 411-417. [Crossref]

36. Liddi R, Beales PE, Rosignoli G, Pozzilli P (2000) Incomplete Freund's adjuvant reduces diabetes in the non-obese diabetic mouse. Horm Metab Res 32: 201-206. [Crossref]

37. Armentero MT, Levandis G, Nappi G, Bazzini E, Blandini F (2006) Peripheral inflammation and neuroprotection: systemic pretreatment with complete Freund's adjuvant reduces 6-hydroxydopamine toxicity in a rodent model of Parkinson's disease. Neurobiol Dis 24: 492-505. [Crossref]

38. Huijbers EJ, Femel J, Andersson K, Björkelund H, Hellman L, et al. (2012) The nontoxic and biodegradable adjuvant Montanide ISA $720 / \mathrm{CpG}$ can replace Freund's in a cancer vaccine targeting ED-B-a prerequisite for clinical development. Vaccine 30: 225-230. [Crossref]

39. Slingluff CL, Petroni GR, Smolkin ME, Chianese-Bullock KA, Smith K, et al. (2010) Immunogenicity for CD8+ and CD4+ T cells of 2 formulations of an incomplete freund's adjuvant for multipeptide melanoma vaccines. J Immunother 33: 630-638. [Crossref]

40. Lai RP, Seaman MS, Tonks P, Wegmann F, Seilly DJ, et al. (2012) Mixed adjuvant formulations reveal a new combination that elicit antibody response comparable to Freund's adjuvants. PLoS One 7: e35083. [Crossref]

41. Awate S, Babiuk LA, Mutwiri G (2013) Mechanisms of action of adjuvants. Front Immunol 4: 114. [Crossref]

42. Yamamoto N (1996) Structural definition of a potent macrophage activating factor derived from vitamin D3-binding protein with adjuvant activity for antibody production. Mol Immunol 33: 1157-1164. [Crossref]

43. Bradstreet JJ, Vogelaar E, Thyer L (2012) Initial Observations of elevated Alpha-nAcetylgalactosaminidase Activity Associated with Autism and Observed Reductions from GC Protein-Macrophage Activating Factor Injections. Autism Insights 4: 31-38.

44. Branca JJ, Morucci G, Malentacchi F, Gelmini S, Ruggiero M, et al. (2015) Effects of oxaliplatin and oleic acid Gc-protein-derived macrophage-activating factor on murine and human microglia. J Neurosci Res 93: 1364-1377. [Crossref]

45. Ruggiero M (2017) A Novel Method to Enhance Immune Responses Induced by HIV DNA Vaccination. [https://bioaccent.org/hiv/hiv27.php]

46. Yamamoto N, Suyama H, Yamamoto N (2008) Immunotherapy for Prostate Cancer with Gc Protein-Derived Macrophage-Activating Factor, GcMAF. Transl Oncol 1: 6572. [Crossref]

47. Ruggiero M, Ward E, Smith R, Branca JJ, Noakes D, et al. (2014) Oleic Acid, deglycosylated vitamin D-binding protein, nitric oxide: a molecular triad made lethal to cancer. Anticancer Res 34: 3569-3578. [Crossref]

Copyright: (C2018 Ruggiero M. This is an open-access article distributed under the terms of the Creative Commons Attribution License, which permits unrestricted use, distribution, and reproduction in any medium, provided the original author and source are credited. 